\title{
Akzeptanz von Pflegerobotern im Krankenhaus
}

\author{
Eine quantitative Studie
}

Ivonne Honekamp, Fakultät für Wirtschaft, Hochschule Stralsund, Zur Schwedenschanze 15, 18435 Stralsund

(ivonne.honekamp@hochschule-stralsund.de), (1) orcid.org/0000-0001-8277-4197

Larissa Sauer, Albertinen Krankenhaus Hamburg (larissa.sauer@gmx.de)

Thomas Wache, FOM Hochschule für Oekonomie \& Management (th.wache@t-online.de)

Wilfried Honekamp, Fachhochschulbereich, Akademie der Polizei Hamburg (wilfried.honekamp@polizei-studium.org), @ orcid.org/0000-0003-2937-7047

Angesichts des Pflegefachkräftemangels, gibt es u. a. den Vorschlag, Pflegeroboter zur Pflege älterer Menschen im Krankenhaus einzusetzen. Es wurden 120 Seniorinnen und Senioren in einer betreuten Wohnanlage zu ihrer Einstellung gegenüber Pflegeroboter-Modellen RIBA, Care-0-bot, Pepper und Paro befragt. Die befragten lehnen Pflegeroboter im Krankenhaus nicht grundsätzlich ab, Akzeptanz und Skepsis hängen aber stark vom Einsatzszenario ab. Wenn Senioren Informationen zu Pflegerobotern erhalten, kann dies Neugier auf neue Technologien wecken. Die große Mehrheit der Befragten geht davon aus, dass Pflegeroboter Pflegekräfte körperlich und zeitlich entlasten können. Es ist anzunehmen, dass Akzeptanz für Pflegeroboter steigen wird, wenn Betroffene in die Technologieentwicklung und in deren Integration in die Pflegepraxis eingebunden werden.

\section{Acceptance of care robots in hospitals \\ A quantitative study}

In view of the shortage of skilled nursing staff, there is a proposal to use nursing robots for elderly people in hospitals. 120 senior citizens in an assisted living facility were interviewed about their attitude towards four selected nursing robots. The models RIBA, Care-0-bot, Pepper and Paro were considered. The questionnaire was based on the Technology Usage Inventory. The results show that senior citizens do not reject nursing robots in hospitals in principle, but that acceptance and skepticism depend strongly on the application scenario. It was observed that the provision of information on care robots can arouse great curiosity among seniors regarding new technologies. The vast majority of senior citizens surveyed assume that nursing robots can relieve nursing staff physically and temporally. It can be assumed that acceptance will be all the higher the more those affected will be involved in the development and integration of the technology into existing processes.

This is an article distributed under the terms of the Creative Commons Attribution License CCBY 4.0 (https://creativecommons.org/licenses/by/4.0/)

https://doi.org/10.14512/tatup.28.2.s58

Submitted: 21. 01.2019. Peer reviewed. Accepted: 02.05.2019
Keywords: technology acceptance research, nursing robots, service robots, hospital, residential community

Das deutsche Gesundheitswesen leidet schon heute an einem Pflegekräftemangel, der sich in Zukunft noch verstärken wird (König 2017). Daher befassen sich WissenschaftlerInnen und PolitikerInnen seit einigen Jahren mit Maßnahmen, um auch in Zukunft eine qualitativ hochwertige Pflege in Krankenhäusern und in der Altenpflege sicherstellen zu können. Hierzu zählen u. a. das Pflegestellenförderprogramm (2016-2018) des Bundesministeriums für Gesundheit, die Akademisierung der Pflegeberufe, die Vereinheitlichung der Ausbildung der Pflegeberufe, die Aufwertung von pflegeintensiven Fallpauschalen oder die Anwerbung von Pflegekräften aus dem Ausland und die Pflegepersonaluntergrenzen. Die Erforschung und Entwicklung neuer assistiver Technologien zur Unterstützung der Alten- und Krankenpflege bieten ein weiteres Potenzial, und es ist anzunehmen, dass Fördermittel für diese Technologien zukünftig steigen werden.

Pflegeroboter eignen sich neben dem Einsatz in der Altenpflege auch für den Bereich der Krankenpflege in Krankenhäusern. In Zukunft wird der Anteil der über 65-jährigen PatientInnen im Krankenhaus stark ansteigen, da ältere multimorbide Personen häufiger stationär versorgt werden müssen als jüngere Personen. Zudem haben ältere PatientInnen zumeist Begleiterkrankungen, die nach Einlieferung ins Krankenhaus zu langen Liegezeiten führen können (Schnoor et al. 2011). Dieser Beitrag konzentriert sich daher auf die Untersuchung der Einstellung von SeniorInnen gegenüber dem Einsatz von Robotern in Krankenhäusern. Ziel der Untersuchung war es, mit Hilfe einer schriftlichen Befragung Hinweise zu generieren, unter welchen Bedingungen und in welchen Situationen SeniorInnen dem Einsatz von Pflegerobotern in Krankenhäusern zustimmen oder ablehnen. Dieses Wissen kann dazu genutzt werden, die Betroffenen im Rahmen der bedarfsorientierten Forschung und Entwicklung zu neuen Technologien im Krankenhaus einzubinden. 
Hierzu wurden SeniorInnen hinsichtlich ihrer Einstellung gegenüber dem Einsatz verschiedener Roboter und möglicher Einsatzszenarien im Krankenhaus befragt.

\section{Akzeptanz von Pflegerobotern}

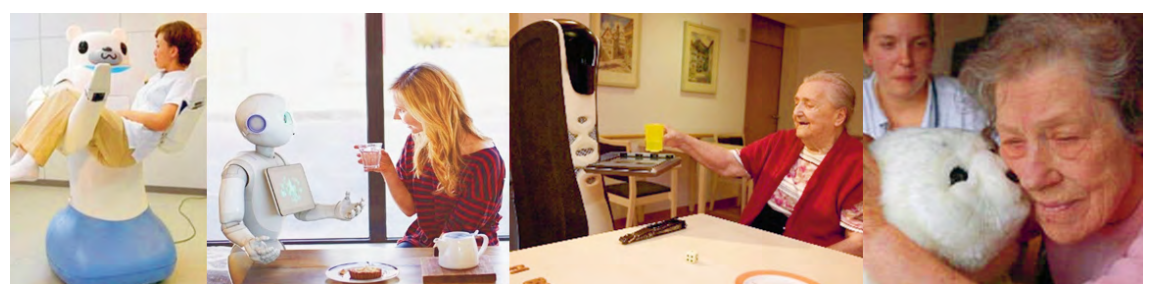

Abb.1: Anwendungsbeispiele im Fragebogen zu den Robotern RIBA, Pepper, Care-0-bot und Para.

Quellen: Salton 2009; Kühn 2017; Kilian 2012; Ernert 2017
Bislang konzentrieren sich wissenschaftliche Untersuchungen zur Akzeptanz von Pflegerobotern auf den Bereich der Altenpflege in stationären Pflegeeinrichtungen sowie, unter der Bezeichnung Ambient Assisted Living, im häuslichen Umfeld (Jacobs und Graf 2011; BMBF 2015; Meyer 2011). Der Einsatz von Prototypen verschiedener Pflegeroboter wurde unter reellen Bedingungen bislang nur in Pflegeheimen hinsichtlich Akzeptanz und Nutzen sowohl auf Seiten der BewohnerInnen als auch auf Seiten der Pflegekräfte untersucht (Hogreve et al. 2011; Meyer 2011).

Befragungen zur Akzeptanz von neuen Technologien werden in der Regel entweder durch repräsentative Studien und Fokusgruppeninterviews oder im Rahmen von Modellprojekten durchgeführt (Hennen 2002; Hindriks et al. 2018). Künemund (2015) stellt fest, dass repräsentative Studien zu Technikinteresse, -akzeptanz und -nutzung eher zu dem Ergebnis kommen, ältere Menschen stünden Technologien generell weniger aufgeschlossen und eher skeptisch gegenüber. Werden Befragungen dagegen im Rahmen von Begleitforschungen zu konkreten Projekten durchgeführt, würde das Interesse älterer Menschen an technischen Assistenzsystemen eher hoch sein.

Diese Unterschiede können durch verschiedene Faktoren verursacht worden sein. So wird den Befragten in repräsentativen Studien in der Regel ein konkretes Szenario beschrieben, in welches sich die TeilnehmerInnen hineinversetzen müssen, um zu beurteilen, inwieweit sie zukünftig diese Technologien nutzen oder ihnen vertrauen würden. Es hängt somit davon ab, welches Anwendungsszenario die Untersuchungsleitung wählt, wie sie es beschreibt und wie gut sich die Befragten in es hineinversetzen können. Die Befragten einer repräsentativen Studie sind möglicherweise anhand der abstrakten Beschreibung des Szenarios gar nicht in der Lage, sich in eine Anwendungssituation hineinzuversetzen und sich den konkreten Nutzen vorzustellen (Künemund 2015). Kommt eine Person dagegen im Rahmen eines Modellprojektes direkt mit der neuen Technologie in Berührung und erfährt selbst einen möglichen Nutzen dieser Technologie, kann sich das Antwortverhalten hin zu mehr Akzeptanz ändern. Jedoch sind auch Befragungen im Rahmen der Begleitforschung mit Problemen behaftet, die die Aussagekraft beeinflussen. Zum einen sind die Stichproben in der Regel sehr gering und zum anderen hat die Selektivität der Stichprobe möglicherweise einen Einfluss auf das Ergebnis von Befragungen. Da anzunehmen ist, dass eher technikaffine SeniorInnen an Modellprojekten teilnehmen, werden diese eine höhere Technikakzeptanz aufweisen als der Durchschnitt aller SeniorInnen.
Betrachtet man Befragungen, in denen es nicht um ein allgemeines Technikinteresse von SeniorInnen geht, sondern um die Nutzung neuer Technologien im Rahmen des altersgerechten Wohnens, entsteht ein zunächst überraschendes Bild. So kommen Studien von Claßen (2012) sowie Kruse und Schmitt (2015) zu dem Ergebnis, dass ältere Menschen diesen Technologien weniger reserviert gegenüberstehen und auch weniger Berührungsängste haben als oft angenommen. Akzeptanz von Technologien im Rahmen des altersgerechten Wohnens lässt sich womöglich damit erklären, dass viele SeniorInnen bereits einen konkreten Unterstützungsbedarf haben, der durch neue Technologien beantwortet wird. Konkrete Auseinandersetzung mit assistiver Technologie in der Pflege sowie konkreter Nutzen scheinen die Akzeptanz zu steigern.

\section{Methodik}

Voraussetzung für den erfolgreichen Einsatz von Pflegerobotern im Krankenhaus ist zum einen die an Nutzerbedürfnissen und -anforderungen orientierte Entwicklung neuer Technologien und zum anderen die Akzeptanz auf Seiten der NutzerInnen. Zu den NutzerInnen im Krankenhaus zählen zum Beispiel Pflegekräfte, ÄrztInnen, PatientInnen und deren Angehörige. In dieser Arbeit liegt der Fokus auf der Nutzergruppe der PatientInnen, wobei hier wiederum die SeniorInnen im Speziellen betrachtet werden.

Im Rahmen dieser Studie wurden vier Pflegeroboter betrachtet, die in Zukunft vermehrt in Krankenhäusern eingesetzt werden könnten, um die Pflegekräfte zu entlasten. Hierbei handelt es sich um den Pflegeroboter RIBA bzw. ROBEAR, welcher 2015 als Nachfolgemodell von RIBA eingeführt wurde, den Roboter Pepper, den Care-O-bot und die Robbe Paro. Mit dem Ziel, Akzeptanz und Skepsis gegenüber diesen Robotern in unterschiedlichen Einsatzszenarien zu untersuchen, wurden eine schriftliche und bebilderte Erklärung der Funktionsweise einzelner Roboter sowie eine schriftliche Befragung gewählt, auch wenn die abstrakte Beschreibung des Szenarios die TeilnehmerInnen nach Künemund (2015) möglicherweise nicht in die Lage versetzt, sich die konkrete Interaktion bzw. den konkreten Nutzen vorzustellen. Eine Alternative wäre die direkte Konfrontation mit den Robotern gewesen, was jedoch aufgrund der begrenzten Ressourcen in dieser Untersuchung nicht möglich war. Eine Klärung von Verständnisfragen war aufgrund der gewählten Befragungsmethode ebenfalls nicht möglich. 
Es wurde ein Fragebogen mit einer geschlossenen Fragenstruktur mit insgesamt 30 Items auf Grundlage des Technology Usage Inventory (Kothgassner et al. 2013) erstellt. Einleitend zum Frageteil wurden für jeden Roboter eine Beschreibung und Bilder mit möglichen zukünftigen Anwendungsbeispielen bereitgestellt - bis auf die Robbe Paro befinden sich die genannten Pflegeroboter noch in der Entwicklung. Bei RIBA wurde auf sein Potenzial hingewiesen, Menschen mit seinen gepolsterten Armen zu mobilisieren. Für Pepper wurde beschrieben, wie er zukünftig individuelle Menschen, Mimik und Gestik erkennen und mit Bewegungen und Sprache darauf reagieren soll. Es wurde weiterhin dargestellt, dass auf seinem Display Spiele und Informationen bereitgestellt werden können und dass Pepper Menschen Gesellschaft leisten kann. Bezüglich Care-O-Bot wurde den TeilnehmerInnen erklärt, dass er Getränke und Medikamente bringen könne. Paro wurde als flauschige Robbe beschrieben, die zu therapeutischen Zwecken eingesetzt wird. Sie könne beruhigend wirken, den Zugang zu z. B. dementiell erkrankten Menschen erleichtern und reagiere auf das Streicheln durch einen Menschen mit Bewegungen von Schwanz, Kopf und Augen.

Als Studienpopulation wurden BewohnerInnen einer betreuten Service-Wohnanlage im Norden Hamburgs gewählt, die laut Aussage der zuständigen Leitung alle 60 Jahre oder älter waren und mindestens einen Krankenhausaufenthalt erlebt hatten. Von etwa 160 Menschen in der Wohnanlage waren laut Leitung etwa 120 BewohnerInnen sowohl kognitiv als auch körperlich in der Lage, an der Umfrage teilzunehmen. In dem dreiwöchigen Erhebungszeitraum vom 19. März 2018 bis 09. April 2018 wurden 102 der 120 verteilten Fragebögen über eine aufgestellte Box in der Eingangshalle des Wohnbereiches abgegeben. Davon waren drei Fragebögen nicht vollständig ausgefüllt, so dass 99 von 120 Fragebögen zur Auswertung verwendet werden konnten. Dies entspricht einer Rücklaufquote von $82,5 \%$.

\section{Ergebnisse}

Die durch die Auswertung der Fragebögen erhaltenen Daten werden im Folgenden dargestellt und beschrieben. Dabei werden erst die Antworten bezüglich der einzelnen Modelle der Pfle- geroboter und deren verschiedene Tätigkeiten differenziert betrachtet. Am Ende wird auf die Akzeptanz bzw. Skepsis der Befragten eingegangen.

\section{Pflegeroboter RIBA}

Abbildung 2 zeigt die Antworten der Befragten auf die fünf Fragen zum Pflegeroboter RIBA. Ein Vergleich der ersten zwei Fragestellungen zeigt, dass sich 73 der Befragten (73,7\%) mit Hilfe und 26 (26,3\%) ohne Hilfe einer Pflegekraft von RIBA aus dem Bett mobilisieren lassen würden. Bei der dritten Frage gaben 60 Bewohner $(60,61 \%)$ an, dass sie Schwierigkeiten hätten, RIBA bei der Mobilisierung aus dem Bett zu vertrauen. Etwas mehr als die Hälfte der Befragten glauben, dass der Einsatz von RIBA Gefahren für sie birgt. 54,5\% lehnen die Vorstellung ab, dass die Assistenz von RIBA ihren Krankenhausaufenthalt angenehmer machen könnte. 


\section{Fragen zu der allgemeinen Neugierde, Interesse und Nützlichkeit}

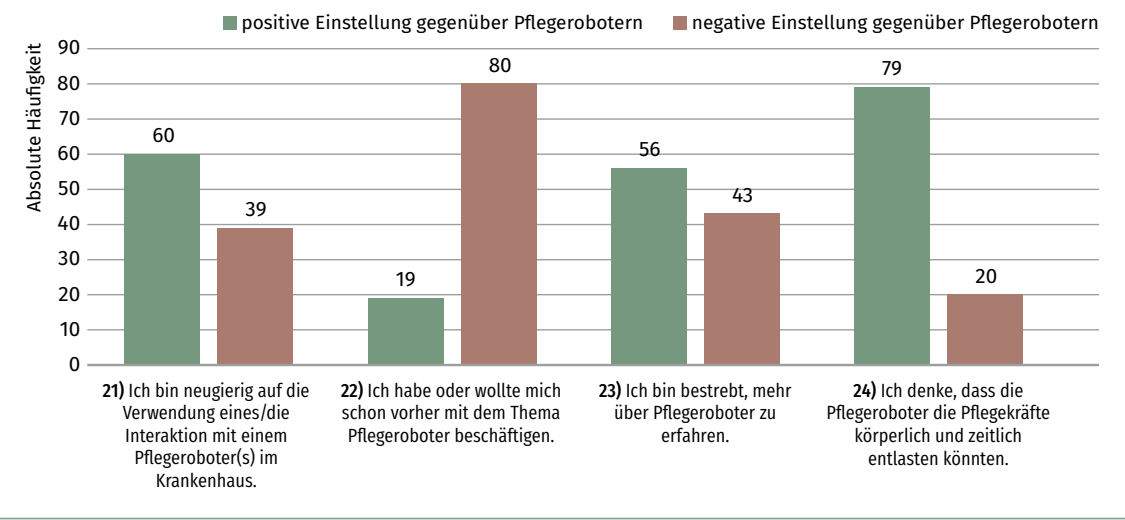

Abb.4: Fragen zu der allgemeinen Neugierde, Interesse und Nützlichkeit.

Quelle: Eigene Darstellung

\section{Fragen zu der allgemeinen Ängstlichkeit und Skepsis}

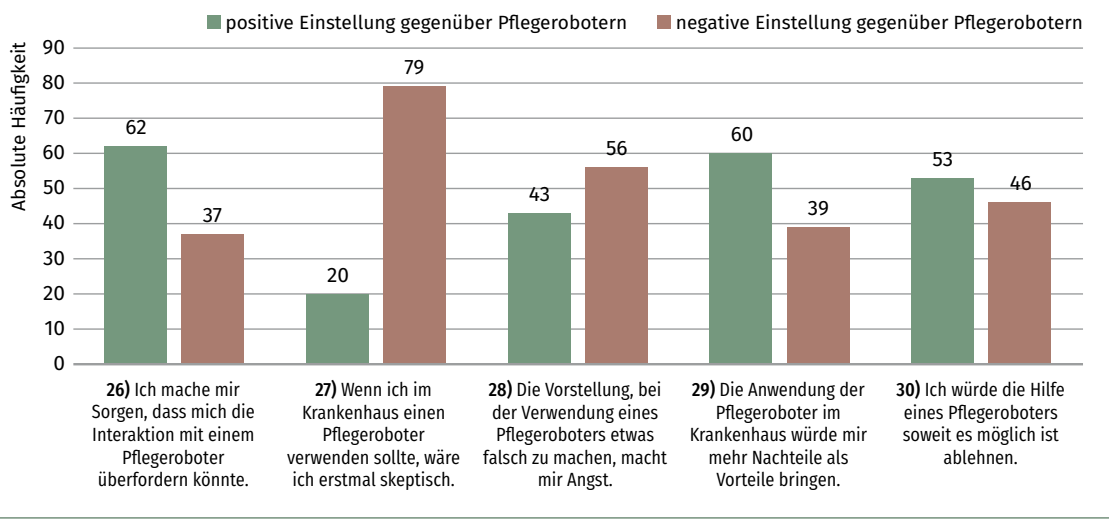

Abb.5: Fragen zu der allgemeinen Ängstlichkeit und Skepsis.

\section{Pflegeroboter Pepper}

Die Nutzung des Roboters im Rahmen eines Gesprächs oder zum Zeitvertreib wurde durch 66 Befragte $(66,7 \%)$ abgelehnt und negativ bewertet. Im Gegensatz dazu würden 53 Befragte $(53,5 \%)$ sich von Pepper aufklären und informieren lassen. Etwa die Hälfte der Befragten würde den Informationen von Pepper vertrauen. 69,7\% sehen keine Gefahr in der Verwendung von Pepper. 41,4\% glauben, dass dieser Pflegeroboter ihnen den Krankenhausaufenthalt angenehmer machen würde.

\section{Pflegeroboter Care-0-bot}

Dreiviertel der Befragten (74,7\%) würden sich von Care-O-bot Getränke oder Lebensmittel bringen lassen. Aber nur 28,3\% würden Medikamente vom Pflegeroboter an- und einnehmen. 35 Befragte $(35,4 \%)$ haben Probleme, Care-O-bot bei der Versorgung mit Lebensmitteln und Medikamenten zu vertrauen und 43,3\% denken, dass der Roboter eine Gefahr für sie darstellen könnte. Der Aussage, dass Care-O-bot den Krankenhausaufenthalt angenehmer machen könnte, stimmten 48,5\% zu.

\section{Pflegeroboter Paro}

Die Mehrheit der Befragten kann sich weder vorstellen, dass Paro zur Beruhigung vor einer OP beiträgt $(66,7 \%)$, noch dass er eine unterhaltende Wirkung hat $(62,6 \%)$. Jedoch denken 70 Befragte (70,7\%), dass die Verwendung bei dementiell erkrankten Menschen im Rahmen der Beruhigung und Unterhaltung keine Gefahren birgt. Von den Befragten hätten 53,5\% Schwierigkeiten, sich auf Paro einzulassen. $33 \%$ der Befragten denken, dass die Roboter-Robbe einen Krankenhausaufenthalt angenehmer machen würde.

Abbildung 3 fasst die Ergebnisse hinsichtlich der Akzeptanz zwischen den verschiedenen Einsatzbereichen bzw. den Tätigkeiten der Pflegeroboter zusammen. Es wird dargestellt, wie viele Befragte eine bestimmte Tätigkeit durch den Roboter durchführen lassen oder nutzen würden.

Es ist zu erkennen, dass es Unterschiede sowohl in der Höhe der positiven Einstellung gegenüber den einzelnen Modellen an sich gibt als auch zwischen den Tätigkeiten eines Pflegeroboters. Am höchsten ist die Akzeptanz bei Care-O-bot und RIBA bezüglich nicht-medizinischer Assistenzleistungen bzw. der Unterstützung einer menschlichen Pflegekraft, allerdings gibt es bei diesen auch die größte Skepsis hinsichtlich Einsätzen im medizinischen Bereich und hinsichtlich vollautomatisierter Pflegeassistenz. Der Einsatz von Pepper würde von knapp mehr als der Hälfte der Befragten für die Bereitstellung von Informationen akzeptiert. Bei Paro hingegen sind beide Einsatzbereiche wenig positiv aufgenommen worden und wurden abgelehnt.

\section{Allgemeine Akzeptanz bzw. Skepsis}

Abbildung 4 zeigt die Verteilung der Antworten zur allgemeinen Neugierde, Interesse und Nützlichkeit. Die Beantwortung von Frage 22 zeigt, dass sich 80,8\% der Befragten bisher nicht mit dem Thema Pflegeroboter beschäftigt haben. 56,5\% der Befragten sind bestrebt, mehr über Pflegeroboter zu erfahren und $60,6 \%$ sind neugierig auf die Interaktion mit einem Pflegeroboter im Krankenhaus. $80 \%$ denken, dass Pflegeroboter nützlich wären, um Pflegekräfte körperlich und zeitlich zu entlasten.

Neugier auf innovative Technologien in der Pflege und Akzeptanz für technische Unterstützung der Pflegenden stehen im Kontrast zu Äußerungen von allgemeiner Skepsis und Ängstlichkeit (siehe Abb. 5). Die Antworten zu Frage 27 zeigen, dass 79 der 99 Befragten zunächst skeptisch wären, wenn sie im Krankenhaus einen Pflegeroboter nutzen sollten. Die Angst, bei der 
Verwendung etwas falsch zu machen, ist bei 53,5\% der Befragten vorhanden. Dass eine Interaktion mit einem Pflegeroboter eine Überforderung darstellen könnte, denken 37,4\%, und dass die Verwendung eines Pflegeroboters ihnen mehr Nach- als Vorteile bringen würde, denken $39,4 \%$.

Zudem wurde für Variablen, bei denen ein Zusammenhang vermutet wurde, dieser mit Hilfe der Korrelation nach Spearman getestet. So besteht kein signifikanter Zusammenhang zwischen der Variable „neugierig auf die Interaktion mit einem Pflegeroboter in einem Krankenhaus sein“ und der Variable „sich Sorgen darüber machen, dass die Interaktion mit einem Pflegeroboter sie überfordern könnte" (Spearmans Rho: 0,006 0, p-Wert: 0,9529). Ebenfalls nicht signifikant korrelieren die Variablen „erst einmal skeptisch bei der Verwendung von Pflegerobotern zu sein“ und „neugierig auf die Interaktion mit einem Pflegeroboter in einem Krankenhaus sein“ (Spearmans Rho: -0,0363, p-Wert 0,7216). Auch Personen, die Angst haben, bei der Verwendung etwas falsch zu machen, sind nicht signifikant weniger neugierig als Senioren, die keine Angst haben, etwas falsch zu machen (Spearmans Rho: 0,0486, p-Wert: 0,6329). Eine positive Korrelation besteht zwischen der Skepsis und dem Bestreben, mehr über Pflegeroboter zu erfahren (Spearmans Rho: 0.2033, p-Wert: 0,043 5). Demnach sind die Personen, die Pflegerobotern skeptisch gegenüberstehen, ebenfalls bestrebt, mehr über Pflegeroboter zu erfahren.

\section{Diskussion und Schlussfolgerungen}

Ziel dieser Untersuchung war es, mit Hilfe einer schriftlichen und bebilderten Befragung Hinweise zu generieren, unter welchen Bedingungen und in welchen Situationen der Einsatz von Pflegerobotern in Krankenhäusern auf Akzeptanz oder Skepsis von SeniorInnen trifft. Die Ergebnisse können Anregungen dazu geben, neue Technologien im Krankenhaus den Erwartungen der NutzerInnen, in diesem Fall der SeniorInnen, entsprechend zu entwickeln und einzusetzen. Die vorliegende Studie zeigt, dass sich $81 \%$ der Befragten noch nie mit dem Thema Pflegeroboter beschäftigt haben. Hieraus sowie aus dem geringen, oft aus den Medien erhaltenen Vorwissen, lässt sich möglicherweise auch die allgemein hohe Skepsis gegenüber Pflegerobotern erklären $(80 \%)$. Interessant ist jedoch die Erkenntnis, dass Skepsis und auch Angst davor, bei der Verwendung des Roboters etwas falsch zu machen, die Neugier auf eine tatsächliche Interaktion mit einem Pflegeroboter nicht signifikant schmälern. Obwohl der Großteil der befragten SeniorInnen gegenüber Pflegerobotern skeptisch ist und auch vielen die Vorstellung Angst macht, bei der Verwendung etwas falsch zu machen (57\%), ist doch ein großer Anteil sowohl neugierig auf die Interaktion mit den Robotern als auch bestrebt, im Anschluss an die Befragung mehr über Pflegeroboter zu erfahren. Dies lässt vermuten, dass das Vorstellen der Roboter und deren Funktionen im Rahmen der Befragung dazu geführt hat, dass Teile der Befragten jetzt neugierig auf die neuen Technologien sind und sich darüber wei- ter informieren wollen. Zudem äußert rund ein Drittel der Befragten Ängste bzgl. der Nutzung von Paro. Dies könnte auf diffuse Ängste und grundsätzliche Technikablehnung in den für Paro dargestellten Nutzungsszenarien hindeuten.

Bei dem Vergleich der Akzeptanz von verschiedenen Robotermodellen und Einsatzbereichen fällt auf, dass sich die Höhe der Akzeptanz von Pflegerobotern im Krankenhaus je nach Tätigkeit des Pflegeroboters unterscheidet. Die BefragungsteilnehmerInnen halten folgende Tätigkeiten im Krankenhaus für besonders geeignet, um sie durch einen Pflegeroboter ausführen zu lassen: eine Mobilisation mit Pflegekraft durch RIBA, das Bringen von Getränken und Lebensmitteln durch Care-O-bot und das Aufklären und Informieren durch Pepper. Bemerkenswert ist, dass sich zwar rund $74 \%$ der Befragten Getränke und Lebensmittel von Care-O-bot bringen lassen würden, sich jedoch nur ca. $28 \%$ Medikamente von Care-O-bot ans Bett bringen lassen würden. Diese Ergebnisse machen deutlich, wie groß das Misstrauen gegenüber Robotern ist, wenn es um vollautomatisierte Pflege des menschlichen Körpers bzw. um kritische medizinische Versorgung wie die Einnahme von möglicherweise lebenswichtigen Medikamenten geht.

Jeweils knapp etwas mehr als $40 \%$ der Befragten sind der Ansicht, dass RIBA, Pepper oder Care-o-bot ihren Krankenhausaufenthalt in bestimmten Bereichen angenehmer machen könnten. In unserer Interpretation könnte dies daran liegen, dass die Befragten hoffen, neue Technologien würden gestresste Pflegekräfte entlasten, so dass diese wieder mehr Zeit für persönliche Pflege und zwischenmenschliche Interaktion haben. Möglicherweise empfinden die Befragten es auch als angenehmer, für bestimmte Hilfen einen Roboter, anstatt eine gestresste Pflegekraft zu rufen. Unabhängig davon, was die BefragungsteilnehmerInnen zu ihren Antworten bewegt hat, denken $80 \%$, dass Pflegeroboter Pflegekräfte körperlich und zeitlich entlasten können.

Derzeit erlebt erst ein sehr geringer Teil aller SeniorInnen im Rahmen von Modellprojekten neue assistive Pflegetechnologien, in naher Zukunft wird es jedoch mehr Krankenhäuser geben, die die eine oder andere neue Technologie zur Unterstützung einsetzen. Somit ist es wichtig, den Betroffenen Informationen zu neuen Technologien in geeigneter Form zur Verfügung zu stellen. Hier kommen z. B. Veranstaltungen von Seniorenbeiräten der Kommunen, Krankenkassen, Krankenhäuser und Pflegeheimen in Betracht sowie auch themenspezifische Messen, Konferenzen, Artikel in Tageszeitungen oder Berichte im öffentlichen Fernsehen. Wenn möglich, sollte den SeniorInnen im Rahmen der o. g. Veranstaltungen die Möglichkeit gegeben werden, die Technologien selbst zu erleben und zu berühren. Die hier vorliegende Studie hat gezeigt, dass bereits ein bebilderter Fragebogen zu Robotern im Krankenhaus Neugierde weckt und dazu anregt, sich mit dem Thema weiter auseinanderzusetzen. Erste Kontakte zu Robotern, z. B. in Filialen von Cafés oder Kaufhäusern, könnten ebenfalls Akzeptanz und Neugier hinsichtlich des Robotereinsatzes im Krankenhaus steigern.

Nicht außer Acht gelassen werden sollte jedoch, dass es in erster Linie darum geht, durch die partizipative Einbindung von 
Betroffenen in Entwicklungsprojekten akzeptanzfähige und bedarfsorientierte Lösungen zu schaffen. Die differenzierten Einstellungen der befragten SeniorInnen in dieser Studie hinsichtlich Akzeptanz und Skepsis gegenüber Pflegerobotern in unterschiedlichen Nutzungsszenarien sowie das durch die Befragung und die Auseinandersetzung mit Pflegerobotern geweckte Interesse für assistive Pflegesysteme zeigen deutlich die Notwendigkeit weiterer begleitender Forschung bei der Einführung von Pflegerobotern auf.

\section{Literatur}

BMBF - Bundesministerium für Bildung und Forschung (2015): Vom Roboter gepflegt werden? Für jeden Vierten vorstellbar. Online verfügbar unter https://www.bmbf.de/de/vom-roboter-gepflegt-werden-fuer-jeden-viertenvorstellbar-950.html, zuletzt geprüft am 17.04.2019.

Claßen, Katrin (2012): Technik im Alltag. In: Hans-Werner Wahl, Clemens Tesch-Römer und Jochen Philipp Ziegelmann (Hg.): Angewandte Gerontologie. Interventionen für ein gutes Altern in 100 Schlüsselbegriffen. Stuttgart: Kohlhammer, S. 499-506.

Ernert, Mathias (2017): Roboter in der Pflege. Experte fordert „breite gesellschaftliche Diskussion“. Online verfügbar unter http://www.altenpflege-online.net/ Infopool/Nachrichten/Experte-fordert-breite-gesellschaftliche-Diskussion, zuletzt geprüft am 17.04.2019.

Hennen, Leonhard (2002): Positive Veränderung des Meinungsklimas - konstante Einstellungsmuster. Ergebnisse einer repräsentativen Umfrage des TAB zur Einstellung der deutschen Bevölkerung zur Technik. Berlin: Büro für Technikfolgen-Abschätzung beim Deutschen Bundestag.

Hindriks, Koen; Boumans, Roel; van Meulen, Fokke; Neerincx, Mark; Rikkert, Marcel (2018): An interview robot for collecting patient data in a hospital. In: ERCIM News 114, S. 20-21.

Hogreve, Jens; Bilstein, Nicola; Langnicke, Diane (2011): Alter schützt vor Technik nicht? Zur Akzeptanz technologischer Dienstleistungsinnovationen von Senioren. In: Daniel Bieber und Kathleen Schwarz (Hg.): Mit AAL-Dienstleistungen altern. Nutzerbedarfsanalysen im Kontext des Ambient Assisted Living. Saarbrücken: iso-Verlag, S. 32-50.

Jacobs, Theo; Graf, Birgit (2011): Förderung des Wissenstransfers für eine aktive Mitgestaltung des Pflegesektors durch Mikrosystemtechnik. Fazit: Grundlegende Erkenntnisse aus dem Einsatz von Care-o-bot 3 in WiMi-Care. Working Brief 31, Universität Duisburg-Essen. Online verfügbar unter https://www.uni-due.de/imperia/md/content/wimi-care/wb__31_.pdf, zuletzt geprüft am 17.04.2019.

Kilian, Jens (2012): Care-0-bot 3 in a nursing home. Online verfügbar unter https://www.care-o-bot.de/en/care-0-bot-3/download/images.html, zuletzt geprüft am 17.04.2019.

König, Romy (2017): Pflegekräftemangel. „Die Guten stellen zunehmend Bedingungen“. In: kma 22 (10), S. 28-31.

Kothgassner, Oswald et al. (2013): Technology Usage Inventory. Manual. Wien: ICARUS. Online verfügbar unter https://www.ffg.at/sites/default/files/ allgemeine_downloads/thematische\%20programme/programmdokumente/ tui_manual.pdf, zuletzt geprüft am 17.04.2019.

Kruse, Andreas; Schmitt, Eric (2015): Technikentwicklung in der Pflege aus gerontologischer Perspektive. In: TATuP - Zeitschrift für Technikfolgenabschätzung in Theorie und Praxis 24 (2), S. 21-27.

Kühn, Iki (2017): Pepper, Paul und Co. Was können die Roboter im Retail?
Online verfügbar unter https://etailment.de/news/stories/Roboter-paulpepper-handel-20672, zuletzt geprüft am 17.04.2019.

Künemund, Harald (2015): Chancen und Herausforderungen assistiver Technik. Nutzerbedarfe und Technikakzeptanz im Alter. In: TATuP - Zeitschrift für Technikfolgenabschätzung in Theorie und Praxis 24 (2), S. 28-35.

Meyer, Sibylle (2011): Mein Freund der Roboter. Servicerobotik für ältere Menschen. Eine Antwort auf den demographischen Wandel? Berlin: VDE Verlag.

Salton, Jeff (2009): RIBA the friendly robot nurse. Online verfügbar unter https://newatlas.com/riba-robot-nurse/12693/, zuletzt geprüft am 17.04.2019.

Schnoor, Jörg; Daumann, Frank; Kaisers, Udo (2011): Demografie und (Multi-) Morbiditätsentwicklung im Krankenhaus. In: Gesundheitsökonomie und Qualitätsmanagement 16 (5), S. 310-315.

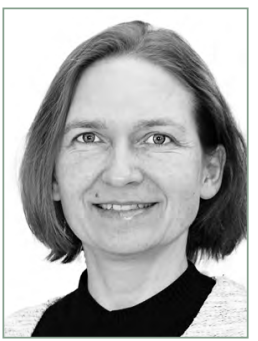

\section{PROF.DR. IVONNE HONEKAMP}

ist Professorin für Betriebswirtschaftslehre, insbesondere Management im Gesundheitswesen an der Hochschule Stralsund. Zuvor war sie Studiengangsleiterin Gesundheit und Management für Gesundheitsberufe an der Carl Remigius Medical School der Hochschule Fresenius, Leiterin des Patientenmanagements in Bundeswehrkrankenhaus Hamburg und vertrat die Professur für Gesundheitspolitik und Gesundheitsökonomie an der Hochschule Zittau/Görlitz.

\section{LARISSA SAUER}

ist Gesundheits- und Krankenpflegerin im Albertinen Krankenhaus Hamburg und Absolventin des Studiengangs Gesundheit und Management für Gesundheitsberufe an der Carl Remigius Medical School der Hochschule Fresenius.

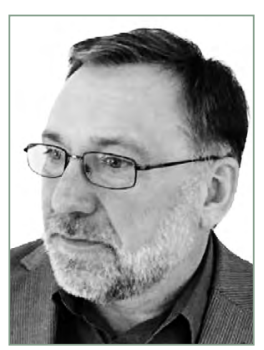

\section{DR. THOMAS WACHE}

ist Dozent an der FOM Hochschule für Oekonomie \& Management und leitet die Physiotherapeutische Praxis am Agaplesion ev. Bathildiskrankenhaus in Bad Pyrmont. Zuvor war er Hochschullehrer im Fachbereich Gesundheit und Soziales der Hochschule Fresenius.

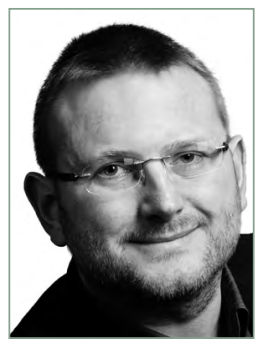

\section{PROF. DR. WILFRIED HONEKAMP}

ist Professor für Angewandte Informatik an der Hochschule in der Akademie der Polizei Hamburg. Davor war er Professor für Softwaretechnik und Programmierung sowie Direktor des HealthCare Management \& Informatics Research Centers an der Hochschule Zittau/Görlitz. 